\title{
Lo comunitario en las radios comunitarias: sentidos en juego
}

The communitary in the communitarian radio: Meanings in the game

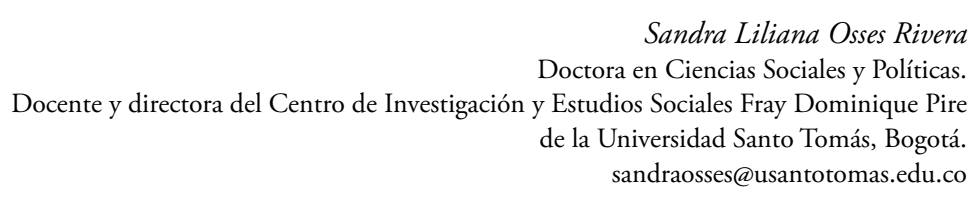

Artículo de investigación

Fecha de recepción: 11 de abril de 2011 • Fecha de aprobación: 27 de abril de 2011

\section{RESUMEN}

¿Qué es lo comunitario de las radios comunitarias? Más allá de las definiciones jurídicas y académicas desde afuera, lo comunitario se construye y se vive dentro de la cotidianidad del trabajo de producción radial en los medios así identificados. Mediante una aproximación antropológica a emisoras comunitarias en México y Colombia, la autora aprehende y documenta el sentido de comunidad subyacente en su programación diaria.

Palabras clave: radio comunitaria, Colombia, México.

\section{Abstract}

What's the community sense in the Latin American community radio? Beyond the legal or academic definitions done outside, the community sense is constructed and lived into the broadcasting production daily work at these media. From an anthropological aproach to the community radio in Mexico and Colombia, the author apprehends and documents the community sense under its everyday programming.

Keywords: Community radio, Colombia, Mexico. 


\section{INTRODUCCIÓN}

Pensar la radio comunitaria en América Latina implica abrir los horizontes posibles de explicación, trascender las fronteras de lo obvio para formular preguntas que permitan dar cuenta del quehacer de los procesos sociales y culturales que subyacen a estos medios, escuchar las voces de los protagonistas y ponerlas en diálogo con las construcciones teóricas que puedan contribuir a su comprensión, ensayar propuestas que permitan asumir su indeterminación y abordar su complejidad, pero sobre todo, implica la comprensión de un fenómeno sociocultural que es expresión de la sociedad en la que vivimos.

Una de las lecciones que extraigo de este trabajo, que ocupó mi labor como investigadora durante siete años, es la de que no existen modelos dados que permitan abarcar la totalidad de un fenómeno social, que no existen las explicaciones últimas y que el llamado al conocimiento es una invitación a hacer de nuestra labor como científicos sociales una tarea de comunicación profunda con el mundo al que asistimos como productores y consumidores de múltiples realidades. Una invitación a hacer visible lo invisible, lo cual significa comprender la construcción del conocimiento como un proceso en el cual un sujeto debe ser capaz de enfrentarse a la realidad para problematizarla, para encontrar lo que se vela ante sus ojos y se niega a ser visto. Una posibilidad de encontrar caminos y abrir a cada paso un nuevo interrogante. Un espacio para renovar la capacidad de asombro y replantear la responsabilidad frente a la producción de pensamiento en una zona como América Latina, donde las realidades se resisten a ser vistas, o los sujetos se resisten a mirarlas de frente.

Este trabajo presenta algunos de los principales resultados de la reflexión investigativa desarrollada entre los años 2000 y 2007 sobre las radios comunitarias en América Latina.

Son necesarias algunas precisiones antes de presentar los resultados: la primera es que se trata de investigaciones abordadas desde una perspectiva socioantropológica; y la segunda, que el trabajo se desarrolló a través de estudios de caso en dos países con una distancia temporal que implica un proceso de reflexión progresivo y marcado por continuos avances y retrocesos, así como por constantes mediaciones entre los marcos conceptuales y las apuestas metodológicas. 
El corpus de análisis se encuentra constituido por seis experiencias, tres estudiadas en Colombia entre el año 2000 y 2002 (tabla 1) y tres casos de radios mexicanas que fueron investigadas entre los años 2004 y 2006 (tabla 2).

Tabla 1. Casos por criterios de selección - Colombia

\begin{tabular}{|l|l|l|l|l|}
\hline \multicolumn{1}{|c|}{ Experiencia } & Contexto & Tiempo de funcionamiento & Situación jurídica & Proyecto comunicativo \\
\hline Suba al aire & Urbano & Antigua & Ilegal & Establecido \\
\hline Vokaribe & Urbano & Antigua & Ilegal & Establecido \\
\hline La Calera F.M. & Rural & Nueva & Legal & Naciente \\
\hline
\end{tabular}

Tabla 2. Casos por criterios de selección - México

\begin{tabular}{|l|l|l|l|l|}
\hline \multicolumn{1}{|c|}{ Emisora } & \multicolumn{1}{|c|}{$\begin{array}{c}\text { Situación } \\
\text { legal }\end{array}$} & $\begin{array}{c}\text { Tamańo de la } \\
\text { población }\end{array}$ & $\begin{array}{l}\text { Tipo de población } \\
\text { predominante }\end{array}$ & \multicolumn{1}{|c|}{$\begin{array}{c}\text { Pertenencia a } \\
\text { organizaciones }\end{array}$} \\
\hline $\begin{array}{l}\text { Radio Ocumicho } \\
\text { (Charapan - Michoacán) }\end{array}$ & Sin licencia & Pequeña & Indígena & Red regional \\
\hline $\begin{array}{l}\text { Radio Erandi } \\
\text { (Tangancícuaro - Michoacán) }\end{array}$ & Con licencia & Intermedia & $\begin{array}{l}\text { Campesina- } \\
\text { semiurbana }\end{array}$ & Red regional - Amarc \\
\hline $\begin{array}{l}\text { La Voladora radio } \\
\text { (Amecameca - Est. De México) }\end{array}$ & Con licencia & Grande & Suburbana & $\begin{array}{l}\text { Amarc - Redes } \\
\text { internacionales }\end{array}$ \\
\hline
\end{tabular}

\section{APUeSTa CONCEPTUAL}

La investigación estuvo orientada por una pregunta tan sencilla como movilizadora: ¿Qué es lo comunitario de las radios comunitarias (RC)? Sobre esta pregunta se avanzó a partir de la comprensión inicial de que las RC son medios de comunicación y como tal deben ser estudiadas como procesos de mediatización. Pero sobre todo, desde la convicción de que en el sustrato de su discurso radiofónico y de su propuesta comunicativa se encuentra la construcción del sentido de lo comunitario que es lo que les confiere su particularidad, orienta su acción colectiva y sustenta la construcción de su identidad social.

Lo que confiere un carácter distintivo a estos medios de comunicación en particular, es la construcción de un sentido de lo comunitario como guía de su desarrollo. La exposición de una hipótesis central, a manera de definición de trabajo para las radios comunitarias, fue operacionalizada en el análisis de los casos de estudio selecciona- 
$\operatorname{dos}^{1}$ y considero que puede ser usada para el análisis de cualquier radio comunitaria con considerable efectividad:

Las radios comunitarias son medios de comunicación, construidos como hechos socioculturales a través de acciones colectivas, que adquieren su sentido de acuerdo con la noción de lo comunitario que construyen emisores, receptores y actores sociales insertos en el mundo social en que se desarrollan, a partir de procesos continuos de comunicación y negociación. Son medios de comunicación producidos por actores que se encuentran en posición de asimetría dentro del sistema comunicacional y social, y son dirigidos a grupos que se identifican con esta colocación dentro de la compleja red de relaciones sociales características del mundo contemporáneo.

El eje de la definición es la construcción de sentido que hacen las radios de ellas mismas como comunitarias y en el análisis se constató que es el punto nodal en la conformación de estos medios de comunicación. De hecho, la pugna por construir un sentido particular de lo comunitario en las emisoras estudiadas constituye el reto más grande que enfrentan sus productores y así lo manifiestan.

Si lo comunitario es una forma significativa de las radios, más que la representación formal de una agrupación social, debemos responder cuáles son esos sentidos que se construyen de lo comunitario. Esos sentidos son detectados de forma muy clara en las funciones comúnmente asignadas a las radios comunitarias por parte tanto de los productores como de los receptores de la misma y de estas quiero resaltar las que surgieron como más determinantes en los casos estudiados. La radio comunitaria cumple la función de:

- $\quad$ ser testigo de la vida de la comunidad local;

- ayudar a rescatar la cultura propia de la comunidad (idioma, costumbres, hábitos, narrativas, etc.);

- servir como espacio de formación y educación;

- consolidarse como espacio de encuentro entre los miembros de la comunidad;

1 En los estudios de caso desarrollados en México. 
- servir como puente para encausar la inconformidad y hacer denuncias públicas;

- tomar el papel de movilizador social;

- publicar la información que los medios de comunicación masiva ocultan o disfrazan;

- desnaturalizar la información que los medios comerciales masifican;

- servir como vínculo entre la comunidad y las instituciones (públicas, privadas, de cooperación, etc.);

- servir como medio de comunicación e información a movimientos sociales, grupos excluidos y todos aquellos que no pueden comunicarse y se encuentran en consonancia con los propósitos del medio;

- promover la cultura local y apoyar su desarrollo, y la lista sigue.

Estas funciones pueden ser organizadas de acuerdo con las propuestas comunicativas que tienen las emisoras y con base en sus procesos de construcción de identidad colectiva para dar cuenta de cuáles son, entonces, los modelos que subyacen a estos medios.

En este documento, me centraré en las "marcas" que considero más importantes en el desarrollo de las radios comunitarias en América Latina, en particular en los países estudiados, las cuales pueden servir como herramientas para su comprensión y para su desarrollo. También pretendo que contribuyan a su desmitificación y que permitan, a partir del proceso de objetivación, asumirlas como procesos históricos y por tanto sujetos al conflicto, a la contradicción y al movimiento.

Aunque sin intentar formular modelos únicos o estáticos que considero del todo impertinentes, presento a continuación una caracterización, a manera de ejercicio analítico, de las concepciones de lo comunitario y la comunidad que subyacen a las radios que participaron de los estudios. Si bien estas no tienen el estatus de teorizaciones macro, sí responden a la condición de ser generalizaciones empíricas que bien pueden arrojar luces al análisis de los medios comunitarios en el mundo contemporáneo. 


\section{ColOMbia: MODELOS EN ACCIÓN, SENTIDOS EN JUEGO}

Los principales hallazgos que emergieron de la investigación realizada en los estudios de caso en Colombia abrieron el camino de la reflexión y permitieron el posterior desarrollo de la definición operativa ya presentada, así como la realización de un análisis comparativo de los procesos de legalización de las radios de América Latina, que no se presentan aquí. De esta manera, se construyeron algunos tipos que buscaron ubicar el sentido de lo comunitario que se construía en las tres emisoras estudiadas así:

- La radio comunitaria como servicio público.

- La radio comunitaria como espacio de construcción de ciudadanía.

- La radio comunitaria como medio de expresión y desarrollo para los municipios.

- La radio comunitaria como medio de comunicación para la inclusión y la visibilización.

La discusión, que aparenta ser formal, entre la selección de uno u otro apellido para las emisoras -comunitarias o ciudadanas-, en realidad determina una construcción de lo público sobre uno u otro paradigma. De hecho, estas relaciones se establecen sobre una concepción de los actores de las radios como operadores de un servicio (concesionarios) o bien como gestores culturales. Y a su vez, estas identificaciones corresponden a formas de racionalidad diferentes que gravitan entre el control y responsabilidad de la autoridad pública y la distribución del poder y la gestión. En la intersección de las dos posturas se puede encontrar la posición de los proyectos comunitarios. Algunos toman partido, otros son conscientes del juego que se establece y entran a negociar desde la perspectiva propia. Las negociaciones no se dan nunca en igualdad de condiciones, por lo cual las estrategias que utilizan los actores se ponen en juego, en función de la relación de poder establecida y pueden transitar desde la conciliación de contenidos, o la contratación de espacios, hasta el recurso a la violencia. Obviamente, esto no se da en el vacío y se determina de acuerdo al contexto y a los otros actores que entran en juego. De esta manera, la radio comunitaria nos permite ver cómo se construye lo público en Colombia a través de la continua disputa entre actores que expresa una forma particular de constitución de lo social. 
A los proyectos que hacen parte de la radio comunitaria en Colombia subyacen proyectos políticos y, en algunos casos, lejos de constituirse en un proyecto centrado en el medio radial es un fenómeno social que emerge como estrategia de los diferentes actores implicados hacia la construcción de proyectos de otro tipo. Como bien lo expresa uno de los actores, "la radio es un pretexto para lograr los objetivos que están en juego".

Lo comunitario adquiere constantemente nuevos sentidos y se determina en los procesos de apropiación que se desarrollan por parte de los diversos actores involucrados. La radio comunitaria, pese a tener un origen que surgió de la movilización social, se transformó en una política de Estado que ha sido reapropiada por grupos gestores de múltiples formas. A su vez, esos grupos se insertan en dinámicas locales y le imprimen un sentido a las políticas que les permite resignificar su función y construir proyectos individuales y colectivos, que no se ubican necesariamente sobre los parámetros de la normatividad.

En estas continuas negociaciones, la comunidad entra como un actor que es convocado y definido de diversas maneras. Así, puede responder a la necesidad de legitimación del proyecto del equipo gestor, ser concebido como un colectivo que requiere orientación, como cliente potencial, capital político, o ser transformado en parte constitutiva del proyecto comunicativo. Cualquiera que sea la opción, el sentido de lo comunitario en los proyectos depende de qué actor le imprime la lógica que lo articula. Es decir, está determinado por las características, intereses y capacidad de apropiación y negociación de los gestores.

\section{MÉXICO, haCia UNA TIPOlOGía DE lo COMUNITARIO EN LAS RADIOS}

Con algunos años de distancia y experiencias diferentes en términos tanto mediáticos como normativos, se proponen algunos tipos para las experiencias estudiadas en México, retomando la definición construida como punto de partida. Por ello, dichos modelos están centrados en el sentido que el colectivo productor asigna a lo comunitario en su propia emisora, que es "lo significado compartido" (Lash, 2000), en relación con la representación que tiene de la comunidad a quien se dirige, la rela- 
ción de la comunidad con el medio $^{2}$, y todo ello sustentado en la forma como dicha comunidad se manifiesta en el espacio local.

\section{La Voladora, una radio ciudadana que vende participación. La comunidad construida}

En La Voladora, lo comunitario responde a una construcción política que se basa en el discurso de los derechos y por tanto gravita sobre el modelo de la radio ciudadana, que propende por una democratización de la comunicación en el mundo actual. Dentro del discurso radiofónico, esta construcción se concreta tanto en las líneas políticas que orientan la programación como en el objetivo explícito de "vender" la participación como un producto.

En La Voladora, la comunidad no responde a una entidad empírica, sino es más una construcción colectiva que trasciende el espacio local, es una comunidad sin rostro a la que los propios productores le confieren ciertas características en un proceso de interacción continua, pero no se trata de una comunidad imaginada sino construida en un proceso de constante observación, diálogo e interacción con el contexto. Por ende, en este modelo no se construyen audiencias, sino se determinan en este modelo no se construyen audicencias, sino se determinan -en palabras de los propios gestores- "públicos meta", comprendidos como interlocutores que se interpelan desde la construcción "objetivada" de quienes producen el medio, es decir, desde las propuestas y compromisos del colectivo gestor con el medio y con la sociedad, un compromiso eminentemente político. Es importante identificar también el hecho de que el propio colectivo se constituye a sí mismo como comunidad, como una comunidad reflexiva en términos de $\mathrm{S}$. Lash, y eso genera un distanciamiento con la audiencia y la población en que se inserta debido a que existe un gran proceso introspectivo. La población percibe al colectivo como externo (percepción reforzada con el origen de la radio), pero a su vez muchos miembros de ella logran insertarse en las dinámicas que propone dentro y fuera del medio; su participación supera lo instrumental o lo técnico y se enraíza en procesos de reconocimiento y visibilización que se traducen

2 Aclaro que el último ítem se construye desde la mirada de los productores, ya que en esta investigación solo se hicieron aproximaciones parciales a los receptores de las radios. 
en la construcción de una identidad compartida por sectores de la población local, pero también por otros actores a nivel nacional y global.

\section{La Preferida, una radio sinfonola que es símbolo de identidad. La comunidad dada}

Las condiciones de esta emisora evidencian una gran dificultad para pensarla como un medio comunitario desde el imaginario y las construcciones que se han hecho de este tipo de medios tanto en el ámbito teórico como práctico, en tanto resultan ser radios que reproducen los esquemas culturales, de comunicación y de poder característicos de medios de comunicación comercial, pero con una gran carencia de recursos y la concentración del poder del medio en una sola persona. No existe en esta radio una propuesta comunicativa intencional y concreta que permita hacer análisis de acuerdo con modelos prefigurados. Sin embargo, desde mi perspectiva, al igual que esta muchas emisoras en México y otros países de América Latina pueden ser pensadas como medios de comunicación comunitaria que se encuentran en proceso de desarrollo. Las condiciones del contexto determinarán en el mediano o largo plazo su capacidad de transformarse en medios más cercanos al ideal comunitario, convertirse en radios comerciales ilegales o desaparecer, pero el uso que la comunidad -esta vez sí la comunidad empírica- hace de ellos es innegable.

En el caso de La Preferida, se trata de una comunidad dada, que usa el medio porque lo necesita y los "altos niveles de audiencia" de que goza en la localidad tienen que ver mucho más con su funcionalidad y con el rol que la emisora ha jugado en el combate al uso indiscriminado de las bocinas o altavoces, que con el discurso que sustenta su producción. Si bien la participación de la comunidad en la producción del medio se limita a aprender la labor de locución, esta dinámica contribuye a la formación de los jóvenes en una comunidad profundamente marginada en la que carecen de opciones educativas y de capacitación.

En este caso no se puede hablar de un proceso de construcción de identidad colectiva de un grupo específico; no obstante, la emisora se ha convertido en un referente de identidad para los habitantes del pueblo, se ha convertido en "un orgullo". En ese sentido podemos afirmar que el hecho de que el $50 \%$ de su programación sea emi- 
tida en lengua purépecha ${ }^{3}$, se dedique mucho tiempo a la programación de música propia y sirva como medio de comunicación básica entre los pobladores de Ocumicho, hace que se cumpla en gran medida con el objetivo enunciado. Las estrategias que se ponen en juego en el desarrollo de la emisora son, en origen, fruto del interés particular de una persona y sin embargo, la propia dinámica de la comunidad indígena hace que el funcionamiento de la radio dependa de las formas de organización y la normatividad tradicional y participe en la vida cotidiana de la comunidad como facilitadora de procesos, además su mera existencia introduce una transformación en la oferta mediática de los habitantes del municipio.

\section{Radio Erandi, una radio para el desarrollo que incorpora la diversidad. La comunidad situada}

El sentido que el colectivo productor de Radio Erandi asigna a lo comunitario tiene que ver con la vinculación de diversos sectores de la población a la producción del medio, con miras a aportar a su desarrollo. El paso del colectivo a la comunidad ha generado en esta radio un proceso de separación entre las esferas administrativa y de producción radial que en muchos casos terminan haciendo que el nivel de apropiación del discurso de lo comunitario entre los miembros tanto del Frente Cívico como del Grupo de Locutores no responda a un significado compartido. Lo que representaría una ventaja en el diseño organizacional de un medio de comunicación institucionalizado, se convierte en un obstáculo en este caso, en términos de la calidad de la propuesta comunicativa de inspiración comunitaria; no obstante, sigue siendo un mecanismo efectivo en la producción del medio.

La propuesta comunicativa de este medio, en atención a la definición de lo comunitario como la apertura de la radio a quienes quieran participar y se comprometan con un programa, deriva en una programación ecléctica que no logra tener un perfil particular, una identidad característica expresada en el discurso radiofónico. Pero, al mismo tiempo, es esa programación reflejo, o mejor, testigo de la diversidad de po-

3 El purépecha es un pueblo indígena asentado principalmente en el estado de Michoacán. De acuerdo con la comisión nacional para el desarrollo de los pueblos indios, en la actualidad las comunidades purépechas están constituidas por más de 200.000 personas que hablan p'urhépecha y comparten su cultura. 
blación de Tangancícuaro. En Radio Erandi muchos géneros musicales, contenidos, voces y mensajes pasan por la programación y muchas personas por el micrófono, la participación en esta radio adquiere sentido en la dinámica local.

Se trata de una comunidad situada, articulada en un territorio en el que construye su identidad y desde allí organiza sus dinámicas de recepción. Así, los programas son escuchados de forma sectorizada y los locutores gozan de popularidad entre aquellas comunidades específicas que comparten su identidad pública y la identidad que en el contenido del programa específico se construye.

El carácter comunitario de las radios se construye de manera intencional en el ámbito de la producción, en tanto construcción de sentido. Es decir, las radios comunitarias definen su especificidad a partir del colectivo que las produce y es en el proceso de la producción en el que se genera el significado compartido de lo comunitario, a partir de una organicidad o funcionalidad del medio con respecto a una entidad preexistente que es denominada comunidad, lo cual deriva en una propuesta comunicativa particular, que es, a su vez, la que diferencia a estos medios de comunicación de otros. Con esto no quiero decir que en la recepción no se configuren también las radios comunitarias, más si las he definido como relaciones comunicativas, sino que el proceso de significación se concreta en una propuesta comunicativa y la construcción de un discurso radiofónico específico que tiene lugar en el proceso de producción.

Lógicamente, a través de sucesivas mediaciones entre producción, recepción y consumo, lo comunitario ingresa en el circuito cultural y constituye el proceso de mediatización. Los receptores de las radios comunitarias, consumen este medio dentro de un abanico amplísimo de ofertas mediáticas, y en su proceso de selección participan criterios que utilizan como marco para su consumo mediático en general. Los receptores, que son denominados por los productores como "la comunidad" en términos genéricos, constituyen una abstracción y al igual que en cualquier proceso de mediatización responden a una representación de quienes formulan la propuesta. Esa comunidad no participa como entidad empírica en el proceso de producción de las radios sino responde a diversas representaciones que los productores tienen de ella y poseen formas diversas de participación que van desde la escucha, pasando por el envío de mensajes o la petición de complacencias y la formulación de quejas o anuncios por medio de la radio, hasta el convertirse en parte de los colectivos gesto- 
res del medio. Esto último, por citar solo un ejemplo, constituye una de las marcas diferenciales de la recepción en las radios comunitarias: el hecho de que los escuchas tengan la opción de ingresar efectivamente a la producción del medio.

\section{Para concluir}

En el desarrollo de la investigación se sugieren caminos diversos, se formulan interrogantes y se abren nuevas líneas de investigación. Para concluir quiero puntualizar en siete de ellos:

- El sentido de las radios comunitarias como procesos políticos, que plantean la necesidad de vincular el surgimiento y desarrollo de medios comunitarios a formas de acción colectiva que les permitan acceder a un sitio propio dentro de las estrategias y políticas culturales de la sociedad actual.

- El estudio de las estrategias, dinámicas y consecuencias de los procesos de legalización de las radios comunitarias en América Latina, que descubre el tema de la relación entre el Estado, las políticas de comunicación y la interculturalidad.

- La condición de marginalidad en la que aún se mantienen los estudios de los medios comunitarios en el campo de las ciencias de la comunicación, que revela una tendencia anárquica del discurso comunitario que termina siendo cómplice de la propia marginalidad y agonía de los proyectos.

- La deuda con la reconstrucción histórica del proceso de la radio comunitaria en América Latina desde una mirada hermenéutica que trascienda los estudios de caso de los cuales, incluso este trabajo, somos víctimas.

- El análisis de radios que se consideran como comunitarias y no cumplen con el modelo prevaleciente y dominante que en casos como La Preferida o La Calera F.M., deben ser contempladas por los grupos y movimientos que apoyan este tipo de medios de comunicación como un reto en términos de su potencialidad, más que ser dejadas de lado por no responder a un modelo ideal o prescriptivo, generando nuevas exclusiones. El desarrollo de experiencias de radio comunitaria que 
surgen en comunidades con altos grados de marginación y con pocos recursos de todo tipo debe ser asumido con una estrategia inclusiva.

- El análisis de los medios comunitarios desde su condición tecnológica, lo cual constituye una demanda urgente si se considera que nuestros países, tarde o temprano, tendrán que entrar en la dinámica de la convergencia. En este contexto, estos medios pueden constituir una alternativa interesante frente a la brecha tecnológica que anuncia más y mayores procesos de exclusión.

- El estudio de las radios comunitarias desde la dinámica de la recepción es una deuda.

- El tema de la sostenibilidad que implica la investigación desde un enfoque de la economía de la comunicación, como herramienta para garantizar la supervivencia y el desarrollo de las radios comunitarias en países con marcos legales restringidos.

En esta América Latina de hoy, signada por el miedo, la incertidumbre y la exclusión, experiencias como las radios comunitarias constituyen espacios que intentan abrir alternativas frente a estos signos: espacios para la solidaridad, la certidumbre, el reconocimiento, la inclusión y la participación. Pero al mismo tiempo, las radios comunitarias pueden convertirse en espacios de exclusión, en procesos que reproducen los esquemas tradicionales de poder, en medios de comunicación muchas veces inconscientes de su capacidad de incidencia, en mitos que no responden a las necesidades de las comunidades en las que se insertan.

Finalmente, quiero dejar sobre la mesa la idea de que las emisoras comunitarias hacen parte de nuevas formas de relación social que surgen como alternativas no solo de comunicación sino de supervivencia dentro del modelo de una sociedad de mercado. Es decir, la supervivencia individual puede ser asumida sobre un proyecto que no reporta beneficios económicos directos pero que apela a la solidaridad y le permite a quienes participan tejer redes de pertenencia y acción que, de una u otra manera, le brinden opciones para sobrevivir. La identidad colectiva puede constituirse para muchos de los actores individuales en un recurso de supervivencia ligado a las políticas del reconocimiento y la visibilización que en el paradigma de la identidad -incluso en lo que hoy se puede denominar las políticas de identidad-pueden ser leídos como 
una salida cultural que permita buscar cómplices en la marginalidad y la exclusión. En este sentido, podremos postular que si bien las radios comunitarias no constituyen una salida de esa misma marginación y exclusión, y no permiten cambiar el sistema, sí abren caminos para ser incluido/s, incluida/s.

En el espíritu que aporta Jesús Martín-Barbero en el texto con que finalizo esta ponencia, es mi deseo -y sé que muchas de las experiencias de radio comunitaria no están lejos de ello- que las radios comunitarias sirvan como espacio de construcción de la indispensable reelaboración simbólica que exige la construcción del futuro en América Latina.

La actual reconfiguración de nuestras culturas indígenas, locales, nacionales, responde hoy especialmente a la intensificación de la comunicación e interacción de esas comunidades con las otras culturas del país y del mundo. Desde dentro de las comunidades, los actuales procesos de comunicación son percibidos a la vez como otra forma de amenaza a la supervivencia de sus culturas -la larga y densa experiencia de las trampas a través de las cuales han sido dominadas carga de recelo cualquier exposición al otro- pero al mismo tiempo, la comunicación es vivida por las comunidades rurales o urbanas como la posibilidad de romper la exclusión, como experiencia de interacción que si comporta riesgos también abre nuevas figuras de futuro. Lo que está conduciendo a que la dinámica de las propias comunidades tradicionales desborde los marcos de comprensión elaborados por los folcloristas y no pocos antropólogos: hay en esas comunidades menos complacencia nostálgica con las tradiciones y una mayor conciencia de la indispensable reelaboración simbólica que exige la construcción de su propio futuro (Martín-Barbero, 2010, p. 297).

\section{REFERENCIAS}

Alfaro, R. M. (1993). Una comunicación para otro desarrollo. Lima: Calandria.

Alfaro, R. M. (2000). Culturas populares y comunicación participativa: en la ruta de las redefiniciones. Razón y Palabra, 18(5). 
Bouissa, Courchet y Orcajo. (1998), Las otras radios. Montevideo: Editorial Nordan Comunidad.

Bourdieu, P. (2000). La miseria del mundo. México: Fondo de Cultura Económica.

Calleja, A. y Solís, B. (2005). Con permiso. La radio comunitaria en México. México: AMARC - AMEDI - Comunicación Comunitaria - CMDPDH.

Cornejo, I. (2002). Apuntes para una historia de la radio indigenista en México. Las voces del Mayab. México: Fundación Buendía.

Chávez, I. (2003). El proyecto de la radio indigenista en el municipio de Cherán, Michoacán, Tesis para optar al grado de Maestra en Antropología Social, CIESAS, México.

Giménez, G. (1998). Cultura, identidad y discurso popular. En Senett y Lameiras (Ed.), El verbo popular. México: El Colegio de Michoacán e Iteso.

Giménez, G. (2005). Estudios sobre cultura e identidad. Libro inédito.

Guber, R. (2004). El salvaje metropolitano: reconstrucción del conocimiento social en el trabajo de campo. Buenos Aires: Paidós.

Gumucio, A. (2001). Haciendo olas: historias de comunicación participativa para el cambio social. Estados Unidos: Fundacion Rockefeller.

Habermas, J. (2000), Facticidad y validez: sobre el derecho y el Estado democrático de derecho en términos de teoría del discurso. Valladolid: Editorial Trotta.

Lash, S. (1997). La reflexividad y sus dobles: Estructura, estética, comunidad. En Beck, U., Giddens, A. y Lash, S., Modernización reflexiva: Politica, tradición y estética en el orden social moderno. Madrid: Alianza Universidad. 
López Vigil, I. (noviembre, 1995). ¿Qué hace comunitaria a una radio comunitaria? Revista Chasqui.

Martín-Barbero, J. (1998). Experiencia audiovisual y desorden cultural. En Mapas nocturnos: Diálogos con la obra de Jesús Martín Barbero. Bogotá: Siglo del Hombre Editores.

Martín-Barbero, J. (2004). Politicas de interculturalidad. Ponencia presentada en el Foro Mundial: Comunicación y Diversidad, Barcelona.

Martín-Barbero, J. (2010). La reinvención patrimonial de América Latina. Sphera Pública. Disponible en http:/www.redalyc.org/src/inicio/ArtPdfRed. jsp?iCve=29719717011.

Matta, M.C. (1995). Investigación radiofónica: de las palabras a los hechos. Quito: UNDA-AL, ALER.

Matta, M.C. (2002). La radio como relación comunicativa. Revista Signo y Pensamiento, 34 (XVIII). Bogotá: Pontificia Universidad Javeriana.

Melucci, A. (1999). Acción colectiva, vida cotidiana y democracia. México: El Colegio de México.

Melucci, A. (2001), Challenging codes. Collective action in the information age. Cambridge: Cambridge University Press.

Rodríguez, C. (2001) Fissures in the mediascape. USA: Hampton Press..

Silverstone, R. (2004). ¿Por qué estudiar los medios? Buenos Aires: Amorrortu Editores.

Soares, R. P. A. y Cabral, Muniz S. (2004). O sequestro da fala Comunitaria. Revista Interscience Place, Rio de Janeiro. 
Sosa, G. (2003). El desarrollo tecnológico de la radio en México y sus implicaciones en la comunicación, tesis de Maestría, UNAM, México.

Winocur, R. (2002). Ciudadanos mediáticos: La construcción de lo público en la radio. Barcelona: Gedisa Editorial.

Yañez, L. (2005). Radio comunitaria, territorio y cultura: aproximación a la comprensión del actual fenómeno, Tesis de Maestría, Universidad de Chile, Santiago. 